\title{
Real-time reservoir flood control operation enhanced by data assimilation
}

\author{
Jingwen Zhang ${ }^{1,2}$, Ximing $\mathrm{Cai}^{2}$, Xiaohui Lei ${ }^{3}$, Pan Liu ${ }^{1}$, Hao Wang ${ }^{3}$
}

${ }^{1}$ State Key Laboratory of Water Resources and Hydropower Engineering Science, Wuhan University, Wuhan 430072, China

${ }^{2}$ Department of Civil and Environmental Engineering, University of Illinois at UrbanaChampaign, Urbana, Illinois, USA

${ }^{3}$ China Institute of Water Resources and Hydropower Research, Beijing 100038, China

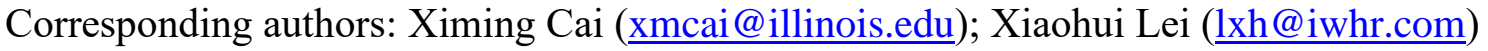

\section{Supplement 1: Preissmann scheme}

Preissmann implicit four-point finite difference scheme is widely used to simulate the streamflow and water level for 1D hydrodynamic model. The difference equations of finite mesh points replace the differential equation of continuous region for simplicity. The derivative of time is the average of forward time derivative for mesh points $j$ and $j+1$, as shown in Eq. S1. The derivative of space is the weighted average of forward space derivative for time steps $t$ and $t+1$, as shown in Eq. S2. In Figure S1.1, point $M$ is the middle of mesh points $j$ and $j+1$ at time period $t+\theta$. The variables at point $M$ can be denoted by the weighted average of the mesh points $j$ and $j+1$ at time periods $t$ and $t+1$, as shown in Eq. S3. The unknown variables of river streamflow and water level at time period $t+1$ for mesh points $j$ and $j+1$ can be determined by the known variables (flow and water level) and the boundary conditions. The weight factor $\theta$ in Eq. S2 and S3 reflects the close degree of the variables at time period $t$ or $t+1$. If the weight factor is closed to 0, the Preissmann method is the explicit finite difference method; otherwise, if the weight factor is closed to 1 , the Preissmann method is the implicit finite difference method. 


$$
\frac{\partial f}{\partial t} \approx \frac{\Delta f}{\Delta t}=\frac{\left(f_{j+1}^{t+1}+f_{j}^{t+1}\right)-\left(f_{j+1}^{t}+f_{j}^{t}\right)}{2 \Delta t}
$$$$
\frac{\partial f}{\partial x} \approx \frac{\Delta f}{\Delta x}=\frac{\theta\left(f_{j+1}^{t+1}-f_{j}^{t+1}\right)+(1-\theta)\left(f_{j+1}^{t}-f_{j}^{t}\right)}{\Delta x}, 0 \leq \theta \leq 1
$$

$f_{M}=\frac{\theta\left(f_{j+1}^{t+1}+f_{j}^{t+1}\right)+(1-\theta)\left(f_{j+1}^{t}+f_{j}^{t}\right)}{2}$

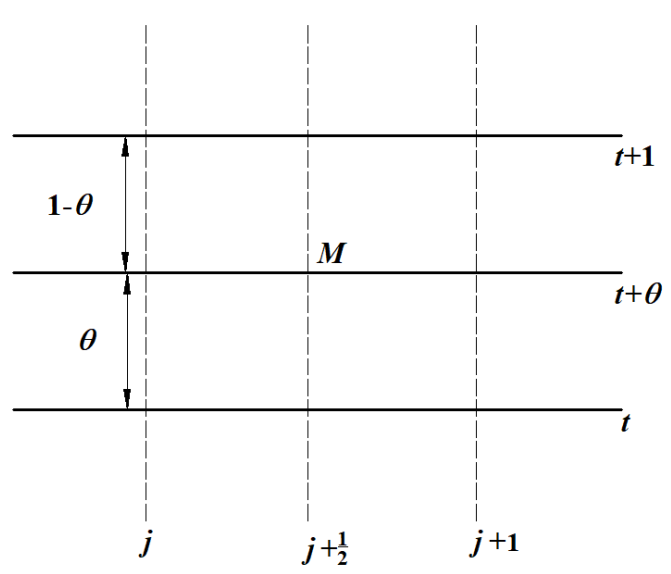

Figure S1.1 Illustration of the Preissmann method

With the difference equations for finite mesh points in Eq. S1 and S2, the Saint-Venant Equations of each sub-river channel can be transformed into Eq. S4. The coefficients can be obtained with the known variables and approximate value of point $M$ (Eq. S3), as shown in Eq.

S5. Since the on-channel reservoir has $N$ channel cross sections, i.e. $N$-1 sub-river channels, the group equation (Eq. S6) denotes all the difference equations of the on-channel reservoir. And it can be solved with known variables and boundary conditions by the chasing method.

$$
\left\{\begin{array}{l}
a_{1 j} Z_{j}^{t+1}-c_{1 j} Q_{j}^{t+1}+a_{1 j} Z_{j+1}^{t+1}+c_{1 j} Q_{j+1}^{t+1}=e_{1 j} \\
a_{2 j} Z_{j}^{t+1}+c_{2 j} Q_{j}^{t+1}-a_{2 j} Z_{j+1}^{t+1}+d_{2 j} Q_{j+1}^{t+1}=e_{2 j}
\end{array}(j=1,2, \cdots, N-1)\right.
$$




$$
\begin{aligned}
& \left\{\begin{array}{l}
a_{1 j}=1 \\
c_{1 j}=\frac{2 Q \Delta t}{\Delta x B_{m}}
\end{array}\right. \\
& e_{1 j}=Z_{j}^{n}+Z_{j+1}^{n}+\frac{1-\theta}{\theta} \times c_{1 j} \times\left(Q_{j}^{n}-Q_{j+1}^{n}\right) \\
& a_{2 j}=2 \theta \times \frac{\Delta t}{\Delta x} \times\left[\left(\frac{Q_{M}}{A_{M}}\right)^{2} \times B_{M}-g A_{M}\right], \\
& \left\{c_{2 j}=1-\frac{4 \theta \Delta t}{\Delta x} \times \frac{Q_{M}}{A_{M}},\right. \\
& d_{2 j}=1+\frac{4 \theta \Delta t}{\Delta x} \times \frac{Q_{M}}{A_{M}}, \\
& e_{2 j}=\frac{1-\theta}{\theta} \times a_{2 j} \times\left(Z_{j+1}^{n}-Z_{j}^{n}\right)+\left[1-4(1-\theta) \times \frac{\Delta t}{\Delta x} \times \frac{Q_{M}}{A_{M}}\right] \times Q_{j+1}^{n}+\left[1+4(1-\theta) \times \frac{\Delta t}{\Delta x} \times \frac{Q_{M}}{A_{M}}\right] \times Q_{j}^{n} \\
& +2 \Delta t \times\left(\frac{Q_{M}}{A_{M}}\right)^{2} \times \frac{A_{\left(x_{j+1}, Z_{M}\right)}-A_{\left(x_{j}, Z_{M}\right)}}{\Delta x}-\frac{2 \Delta t \times g \times n^{2} \times\left|Q_{M}\right| Q_{M}}{A_{M} \times\left(A_{M} / B_{M}\right)^{4 / 3}} \\
& \left(\begin{array}{cccccccc}
a_{0} & c_{0} & & & & & \\
1 & -c_{11} & 1 & c_{11} & & & \\
a_{21} & c_{21} & -a_{21} & d_{21} & & & \\
& & 1 & -c_{12} & 1 & c_{12} & & \\
& \ldots & & \cdots & & \ldots & \\
& & & 1 & -c_{1, N-1} & 1 & c_{1, N-1} \\
& & & a_{2, N-1} & c_{2, N-1} & -a_{2, N-1} & d_{2, N-1} \\
& & & & & a_{N} & d_{N}
\end{array}\right)\left(\begin{array}{c}
Z_{1} \\
Q_{1} \\
Z_{2} \\
Q_{2} \\
\cdots \\
Q_{N-1} \\
Z_{N} \\
Q_{N}
\end{array}\right)=\left(\begin{array}{c}
e_{0} \\
e_{11} \\
e_{21} \\
e_{12} \\
\cdots \\
e_{1, N-1} \\
e_{2, N-1} \\
e_{N}
\end{array}\right)
\end{aligned}
$$




\section{Supplement 2: Parameter calibration}

Roughness coefficients depend on the nature of the channel and streamflow characteristics, reflecting the energy loss due to the friction along the channel and the turbulence in the channel (Aldridge \& Garrett, 1973; Pappenberger et al., 2005). Larger flow resistance results in larger roughness coefficients. The most commonly used method for determining the roughness coefficients is to calibrate against the historical streamflow and water level. The roughness coefficients vary with the topography and the streamflow magnitude. As there are 11 water level observation sections for the on-channel reservoir, the roughness coefficients at 11 sections have been selected as the parameters to be calibrated. The roughness coefficients of other cross sections can be determined by the linear interpolation method.

With the historical flow and water level observations of small and large flood events, the roughness coefficients can be calibrated separately. Constraint Ensemble Kalman Filter with accept/reject method (CEnKF accept/reject) is used as the calibration method (Moradkhani et al., 2005). Figures S2.1 and S2.2 show the calibration of roughness coefficients at 11 observation sections for small and large flood events, respectively. The results demonstrate that the ensemble mean of the roughness coefficients approaches stable values for small and large flood events. The red area, the $90 \%$ confidence interval with the ensemble size of 1000 , shrinks quickly and approaches a stable spread. 

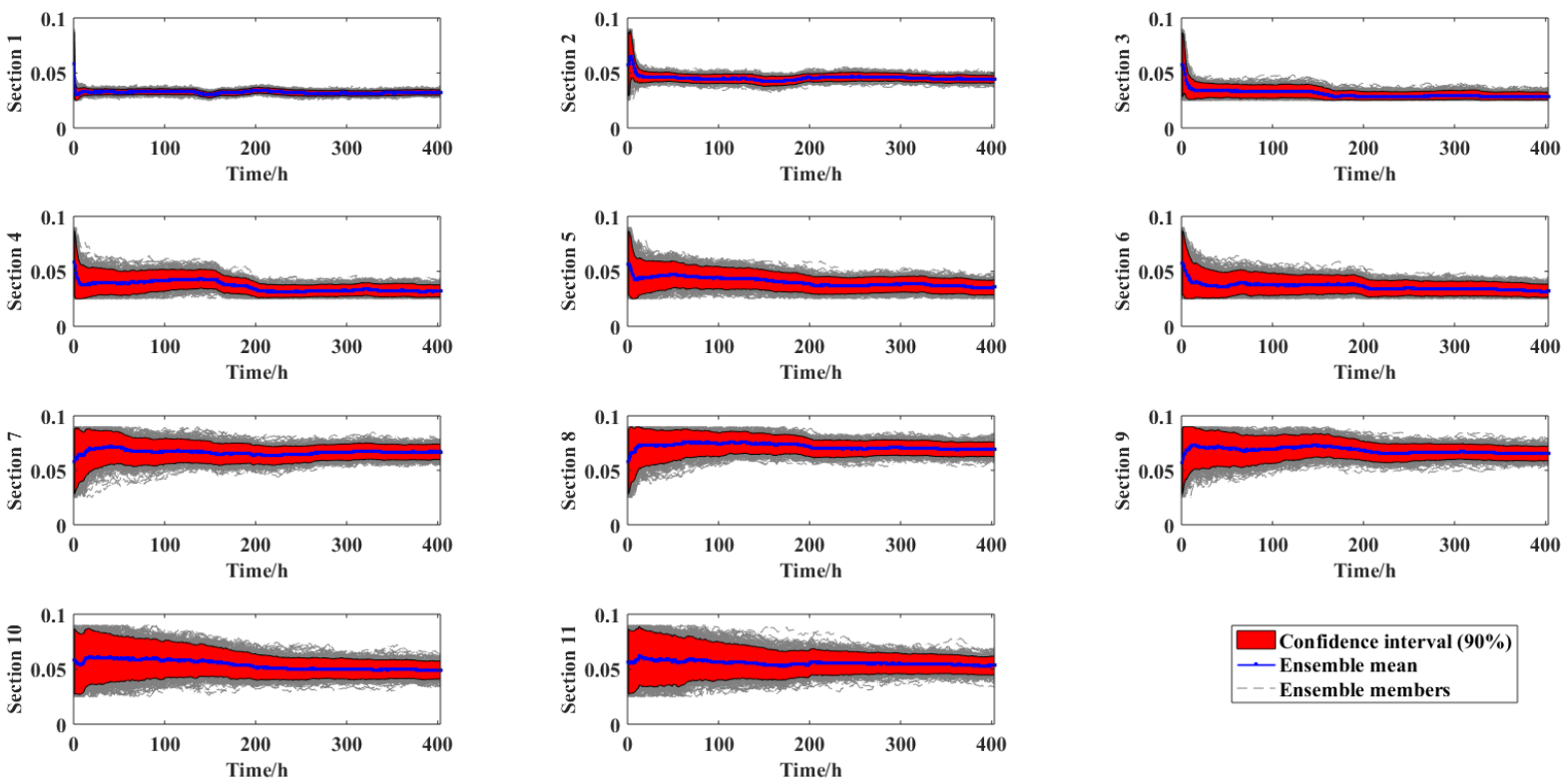

Figure S2.1 Calibration of roughness coefficients at 11 observation sections for small flood event
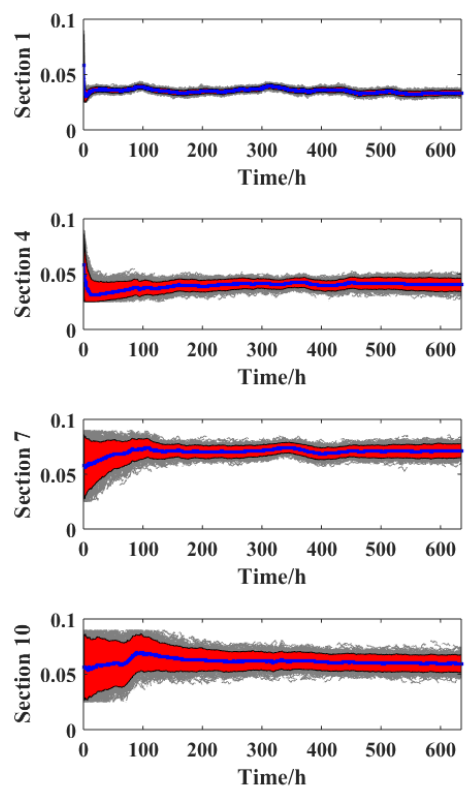
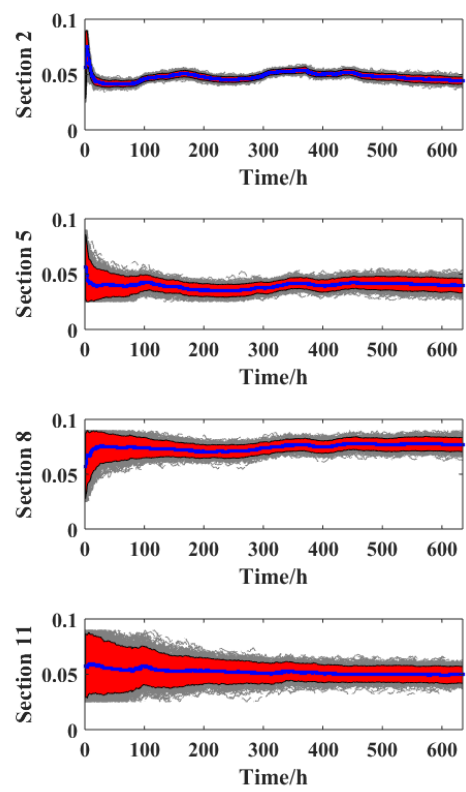
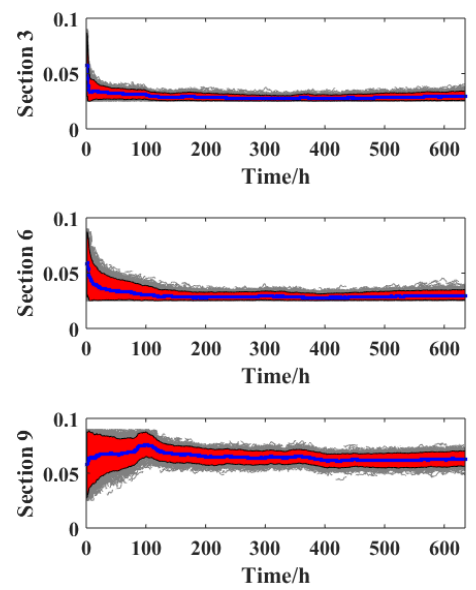

$\square$ Confidence interval (90\%) Ensemble member

Figure S2.2 Calibration of roughness coefficients at 11 observation sections for large flood event

The calibrated roughness coefficients for small and large flood events are determined based on the average value of the ensemble mean for the last 20 time-steps, as shown in Table

S2.1. The calibrated roughness coefficients at the same section is different for small and large flood events, but the difference is acceptable, which is less than 0.01 . 
Table S2.1 The roughness coefficients for small and large flood events

\begin{tabular}{|c|c|c|c|c|c|c|c|c|c|c|c|}
\hline & Section & Section & Section & Section & Section & Section & Section & Section & Section & Section & Section \\
\hline & 1 & 2 & 3 & 4 & 5 & 6 & 7 & 8 & 9 & 10 & 11 \\
\hline $\begin{array}{l}\text { Small } \\
\text { flood }\end{array}$ & 0.0323 & 0.0445 & 0.0286 & 0.0318 & 0.0354 & 0.0320 & 0.0669 & 0.0692 & 0.0655 & 0.0492 & 0.0533 \\
\hline $\begin{array}{l}\text { Large } \\
\text { flood }\end{array}$ & 0.0334 & 0.0446 & 0.0294 & 0.0404 & 0.0400 & 0.0297 & 0.0710 & 0.0771 & 0.0631 & 0.0598 & 0.0499 \\
\hline
\end{tabular}

